\title{
Calculation method to determine capillary properties of building materials with automatic free water intake test
}

\author{
Eero Tuominen ${ }^{1, *}$, and Juha Vinha ${ }^{1}$ \\ ${ }^{1}$ Tampere University, Building Physics, 33720 Tampere, Finland
}

\begin{abstract}
The water absorption coefficient and capillary saturation water content are common building physical material properties. This paper presents a calculation method to determine these values with an automated free water intake test arrangement. Buoyancy affects weighing results in the automatic measurement and it is recommended to take these factors into account when deriving the real water intake of a specimen. A new mathematical method is presented and trial experiments have been conducted. The method is proven to work with the polymer modified plaster, concrete, autoclaved aerated concrete and lightweight aggregate concrete, which represent materials from both extremes of capillary activity. The functionality and advantages of the test arrangement for both low and high suction materials are presented.
\end{abstract}

\section{Introduction}

The transport of liquid water in porous media can be characterized by the liquid water diffusivity which describes the transport coefficient as a function of the moisture state. The liquid water diffusivity can be measured directly [1], but the specimen size is limited, and the equipment needed is expensive.

The well-known and reproducible method to measure water absorption is a free water intake test [2]. The water absorption coefficient $\left(\mathrm{A}_{\mathrm{w}}\right)$ and the capillary saturation moisture content of the specimen $\left(\mathrm{w}_{\text {cap }}\right)$ can be calculated from the test results. These material characteristics are based on unsaturated flow theory, which is proven to be relevant also with building materials [3] [4]. Furthermore, the liquid water diffusivity is based on the same theory and can be estimated by correlation [5] or can be calculated directly [6] from the results of free water intake tests.

The free water intake test can be made by the manual method described in EN ISO 15148 (2002), but the more sophisticated automated method has been developed [8] [9]. This article improves the automated method by representing the calculation method, which eliminates the systematic error caused by buoyancy.

\footnotetext{
*Corresponding author: eero.tuominen@tuni.fi
} 


\section{Measuring methods}

The standard method for determining the water absorption coefficient is described in EN ISO 15148 (2002), NT Build 368 (1991) and ASTM C1585 (2013)-standards. A specimen with regular shape shall be conditioned, dimensions measured, and vertical sides protected against the diffusion and capillary transport of water. Furthermore, other preparations might be needed.

After preparations, one face of the specimen is immersed in water and cumulative water absorption is measured by balance weightings at regular time intervals. Before each weighing, the specimen is cleaned by dampened tissue etc. to remove water from the surfaces. The cumulative water absorption by specimen area is plotted against the square root of time to get a water absorption curve (WAC).

In most cases, WAC has linear part and the slope of the part is described as the water absorption coefficient $\left(A_{w}\right)$. Capillary saturation can instead be calculated only if WAC contains also another linear part like in Fig. 2. Bomberg et al. [2] has presented clear steps for calculation and those are not presented here. For more special pore structures (for example autoclaved aerated concrete, expanded clay aggregate concrete) there are also another calculation methods, for example the one Iannou et al has presented [10].

The biggest source of error in the standard method is the cleaning of the water before weighing and breakdown in water inflow during the weighing. These issues can be avoided by using the automatic measuring device, which is proven to be comparable to the standard method [2].

The automatic device used is composed on the shelf from which can be found own places for balance, parts of a water flow system, water level adjustment tools and a chamber for absorbing water container and specimen holder. The picture of shelf can be seen in Fig. 1.

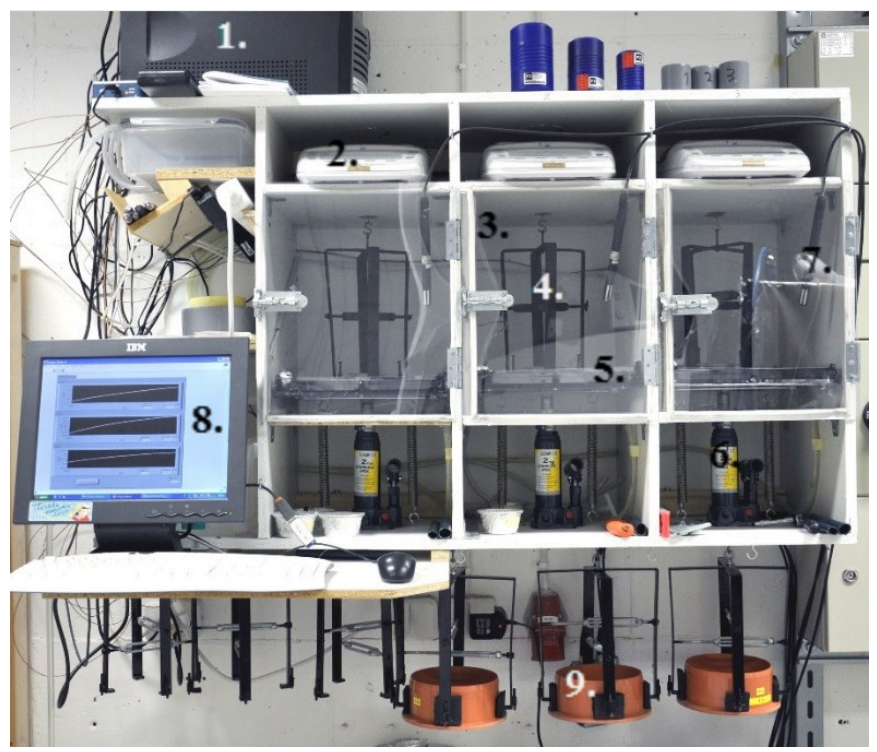

Fig. 1. Automatic measuring device 1. Computer, 2. Balance, 3. Measuring cabinet, 4. Specimen holder, 5. Absorbing water container, 6. Water level adjustment, 7. RH/T sensor, 8. Controlling software, 9. Calibration specimen

The inlets to the measuring cabinet are sealed to minimize evaporation from the water container. With suitable sealing, the relative humidity in the cabinet increases to nearly 100 
$\% \mathrm{RH}$ in less than 15 minutes and a rate of evaporation turns stable. In addition, a small temperature fluctuation of the room where the equipment is located is negligible while the conditions of the measuring cabinet are dominated by an open water surface in the container. Thus, the water temperature needs to settle before an experiment.

The automated method is described [8] and developed [9]. As buoyancy affects the weighing results when the weighing is done during water contact, the instability of the water level causes error in measured WAC. Even small changes in immersion depth causes great differences in balance reading, thus it does depend on specimen size. Also, the change in the temperature of the water affects as the water level changes because of thermal expansion. The effect of buoyancy is found and somewhat perceived [9], but it is not completely eliminated nor described.

\section{Development of the calculation method}

The automatic device automates the weighing process during specimen immersion and the calculation of material characteristic is to be done from WAC similar to the standard method. With automated weighing, the actual WAC need to be calculated from the measured one to avoid systematic error caused by the buoyancy. The calculation method is introduced below.

Buoyancy effects can be calculated if water level is known, but direct measurement of water level by resolution less than $0,01 \mathrm{~mm}$ is not reliable as the surface tension affects to the physical measuring device. Distance-meter based on ultrasound or laser beam could instead be relevant, but an accurate sensor is too expensive in comparison with other measuring devices needed in the equipment. Water level management with overflowing water was also tested, but it was not as reliable as the method described here. Additionally, the overflow and water pumping systems are more vulnerable than standing water.

An outgrowth is to calculate the level changes, which are caused by specimen suction and free water surface evaporation (equation 1). The temperature of the water should be stable to avoid buoyancy effect arising from thermal expansion.

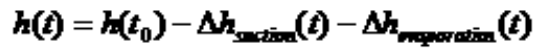

Here $h\left(t_{0}\right)$ is immersion depth at time $t_{0}, \Delta h_{\text {suction }}(t)$ is change in immersion depth caused by specimen suction and $\Delta h_{\text {evaporation }}(t)$ is change in immersion depth caused by evaporation.

The first term, immersion depth $h\left(t_{0}\right)$ at the beginning $t_{0}=0$ can be calculated by balance equation (2), as $\Delta h_{\text {suction }}(t)=\Delta h_{\text {evaporation }}(t)=0$.

$$
\begin{aligned}
& \uparrow N-G+F=0 \\
& \hat{N} \\
& N=m(t) g \\
& G=\left[m_{0}+\Delta m_{r}(t)\right]_{g} \\
& F=\rho_{r} h(t) A_{\text {sociond }} g
\end{aligned}
$$

Here $F$ is buoyancy, $G$ is gravitation, $N$ is supporting force from balance, $m(t)$ is the reading of the balance, $g$ is the acceleration of gravity, $m_{0}$ the dry weight of the specimen, $\Delta m_{w}(t)$ is the mass of absorbed water, $\rho_{w}$ is the density of water, $h(t)$ is defined by function (1), and $A_{\text {specimen }}$ is the area of the face of the specimen immersed in the water. From equation (1) and (2) one can derive $h(0)$ (equation 3).

$$
h(0)=\frac{m_{0}-m(0)}{\rho_{w} A_{m=i-c}}
$$


Value $m(0)$ cannot be specified exactly as $\Delta h_{\text {suction }}(t)$ and $\Delta h_{\text {evaporation }}(t)$ starts running at the time specimen touches the water but first stable balance reading cannot be taken until a few seconds later. It does not matter as only the changes, not the absolute value, in buoyancy is needed.

The second term, change in immersion depth caused by specimen suction are to be calculated next. First, we calculate the volume $\left(V_{\text {absorbed }}\right)$ of water absorbed by the specimen and the change of water level in the water container when the volume volume $\left(V_{w}\right)$ is taken out (4).

$$
\begin{gathered}
\Delta \boldsymbol{v u}_{\mathbf{w}}(\boldsymbol{t})=\boldsymbol{\rho}_{\boldsymbol{w}} \boldsymbol{V}_{\text {cheorbood }}(t) \\
V_{w}(t)=A_{\text {water }} \Delta h_{\text {suction }}(t)
\end{gathered}
$$

Where $A_{\text {water }}$ is area of free water surface. As the volume of water absorbed by the specimen is taken from the water container, we can derive equation (4) and come out with a result (5).

$$
h_{\text {suction }}(t)=\frac{\Delta m_{w}(t)}{\rho_{w} A_{\text {water }}}
$$

The third term, the descend of water level caused by evaporation needs to be calibrated with evaporation experiments. In the experiment the rate of evaporation is measured with circular plastic plates used instead of specimens to avoid suction $\left(\Delta m w(t)=\Delta h_{\text {suction }}(t)=0\right)$. The experimental setup does not differ hence the physics behind equation (2) apply. To separate evaporation experiment from material experiment, we symbolize $m(t)$ as $m_{\text {evaporation }}(t), m(0)$ as $m_{\text {evaporation }}(0)$ and $A_{\text {specimen }}$ as $A_{\text {evaporation }}$ and again merge equations (1) and (2) to derive equation (6).

$$
h_{\text {evaparation }}(t)=\frac{m_{\text {eaporation }}(t)-m_{\text {exaparation }}(0)}{\rho_{w} A_{\text {euparation }}}
$$

The results of evaporation experiments gives us the values $m_{\text {evaporation }}(t), m_{\text {evaporation }}(0)$ and $A_{\text {evaporation. }}$ After several experiments done in various condition one can evaluate equation (6) as a function of evaporation, which express the rate of evaporation by means of water level change.

Now one can calculate the real weight change of the specimen by combining functions (1), (2), (3), (5) and (6). With the solution (equation 7), one can draw the exact WAC.

$$
\frac{\Delta m_{w}(t)}{A_{\text {specimen }}}=\frac{\frac{m(t)-m(0)}{A_{\text {specimen }}}-\frac{\left[m_{\text {eupcration }}(t)-m_{\text {eaparation }}(0)\right]}{A_{\text {evapcration }}}}{\left\{\frac{A_{\text {specimen }}}{A_{\text {water }}}+1\right\}}
$$

\section{Trial experiments}

A functionality of the equipment was tested by ordinary and well-known materials. The materials tested were a polymer modified plaster usually used as a setting coat (plast), autoclaved aerated concrete (aac), expanded clay aggregate concrete (lwac) and concrete (concr). These materials represent both extremes in the capillary activity of common building materials in Finland.

At first the function of evaporation was determined with evaporation experiments done with the plastic plates in diameter 50 and $185 \mathrm{~mm}$. It was in for variation in evaporation rates between experiments. The humidity in the measuring cabinet, the temperature of the cabinet, the water, the measuring room and the humidity of the room was measured and cross-checked one by one if there was correlation to the evaporation rate. The size of the 
plastic plate nor the measured circumstances did not affect the rate of evaporation, but differences in the humidity of the measuring room appeared to correlate to the differences between evaporation rates. The evaporation experiments were performed also between material experiments to confirm the reliability of the function of evaporation.

Several material experiments were done during the development of the equipment and the calculation method. An example of the WAC determined by the final version is shown in Fig.2. The uppermost and dashed line is a measured WAC determined by automatic water intake equipment (the 1 st nominator of eq. 7 ).

The solid line presents the weight chance after it is corrected with the function of evaporation (the nominator of equation 7). It can be seen how the error of evaporation is almost negligible during the first phase of WAC as the measured water absorption coefficient of the concrete specimen measured is relatively high. Later it is increasing gradually as it is proportional to the time and here the $\mathrm{x}$-axis is square root of time.

The lowest curve represents the actual WAC of the specimen as it is calculated from the solid line by correcting the change in immersion depth caused by the specimen suction (whole equation 7). There can be seen also two manual weighing points which are made after the specimen is taken out from the automatic device. The manual measurements are made to ensure the functionality of the calculation method.

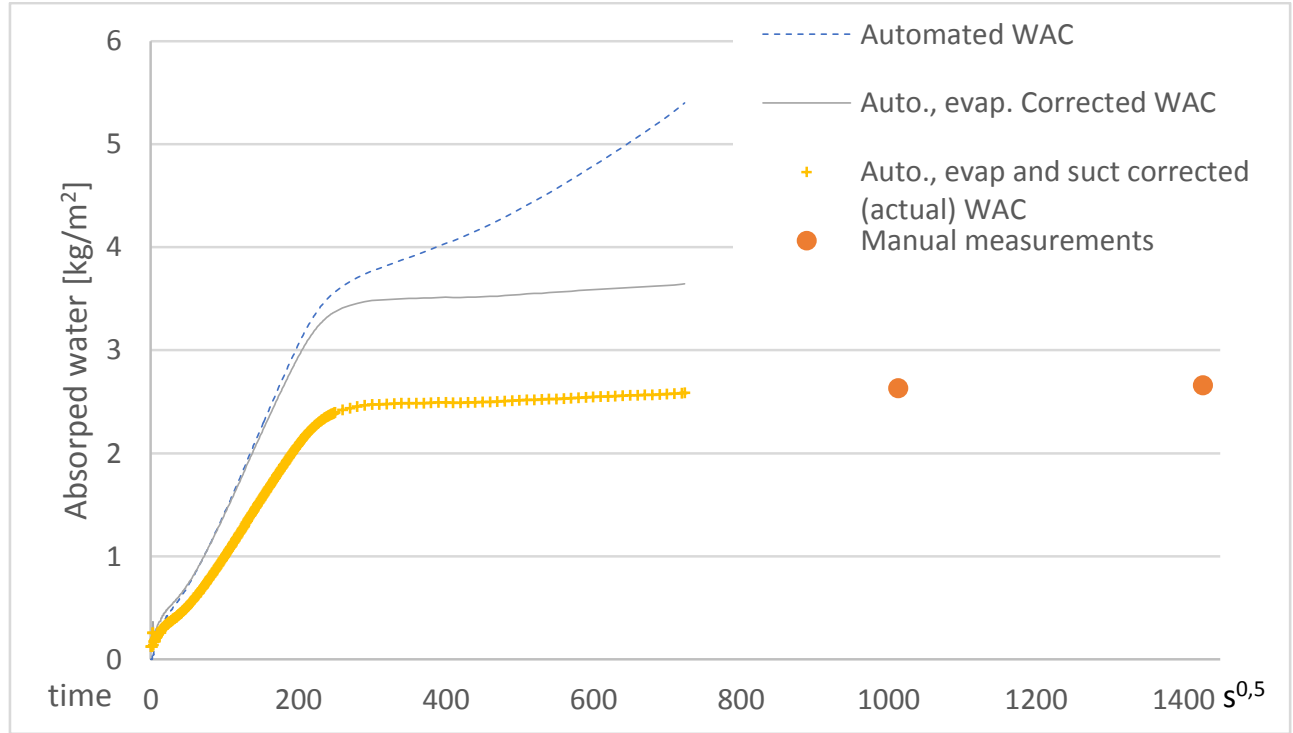

Fig. 2. Automatically and manually measured, evaporation fixed, suction fixed and deduced data of concrete specimen

Before making the linear regression to calculate the water absorption coefficient, the amount of data should be deducted to for example 1 point per $1 \mathrm{~s}^{2}$ as originally there are more data points for each square root of second in the end than at the beginning of the test. The results of material experiments done with the final version of the equipment and the calculation method are shown in table 1.

As the calculation is done by the method presented in [2], the times steps "initial time" $\left(t_{0}\right)$, "final time" $\left(t_{1}\right)$ and "time when the increase of mass is stabilized to a small, constant value for the determination of the capillary moisture content" $\left(t_{2}\right)$ are also presented.

Table 1. Water absorption coefficient, capillary saturation, $10 \%$ confidence interval of given material characteristics and time steps used in calculation (average of all specimens). 


\begin{tabular}{|c|c|c|c|c|c|c|c|}
\hline & $\begin{array}{c}\mathbf{A}_{\mathbf{w}} \\
{\left[\mathrm{kg} / \mathrm{m}^{2} \mathrm{~s}^{{ }^{5}}{ }^{2}\right.}\end{array}$ & \pm & $\begin{array}{c}\mathbf{W}_{\mathbf{c a p}} \\
{\left[\mathrm{kg} / \mathrm{m}^{3}\right]}\end{array}$ & \pm & $\begin{array}{c}\mathbf{t}_{\mathbf{0}} \\
{[\mathrm{s}]}\end{array}$ & $\begin{array}{c}\mathbf{t}_{\mathbf{1}} \\
{[\mathrm{s}]}\end{array}$ & $\begin{array}{c}\mathbf{t}_{\mathbf{2}} \\
{[\mathrm{s}]}\end{array}$ \\
\hline Plast & 0,0012 & 0,0002 & - & - & 450 & 1500 & - \\
\hline $\begin{array}{c}\text { Concr } \\
\mathbf{C 3 2} / \mathbf{4 0}\end{array}$ & 0,010 & 0,001 & 135 & 4 & 70 & 250 & 274 \\
\hline $\begin{array}{c}\text { Concr } \\
\text { C20/25 }\end{array}$ & 0,014 & 0,001 & 112 & 4 & 64 & 150 & 173 \\
\hline AAC & 0,037 & 0,004 & - & - & 53 & 88 & 16 \\
\hline LWAC & 0,048 & 0,016 & 198 & 12 & 4,5 & 9 & 104 \\
\hline
\end{tabular}

\section{Conclusions}

The free water intake test made by the automated device is further developed by calculating the actual weight change curve from the measured one. The changes of water level need to be acknowledged as the buoyancy is affecting the weighing results. Though the effect of evaporation is low in high suction cases, the water level changes as the specimen itself suck the water.

The automated method works best with high suction materials, where the first phase of WAC is limited to one day. With these materials, the actual weight change is defined precisely as the uncertainty of evaporation is low and the biggest sources of errors from the manual method is avoided. When the suction continues more than 3 days, it is recommended to continue measurement with the manual method. The errors of the manual method remain negligible as weighing's are done rarely, once a day or more seldom.

The automated method produces precise data to be used in the inverse modelling of material characteristics. In the case, the initial period of specimen suction could be measured even more accurate if the water container were put on a scale. In the present state, the device alone provides possibility not only to wet the specimen but also dry the specimen in controlled environment.

This study was funded by the Tampere University of Technology core funding. The development of an equipment was partially funded by Kiilto Oy, whose materials were also investigated.

\section{References}

1. J. Carmeliet, H. Hens, S. Roels, O. Adan, H. Brocken, Z. Pavlik, R. Cerny, C. Hall, K. Kumaran, L. Pel. J. Therm. Env. Build. Sci. 27 277-305 (2004)

2. M. Bomberg, M. Pazera, R. Plagge. J. Therm. Env. Build. Sci. 28 227-243 (2005)

3. C. Hall. Mag. Concrete Res. 41 51-61 (1989)

4. C. Hall. Mag. Concrete Res. 37 378-385 (2007)

5. M. K. Kumaran. J. Therm. Env. Build. Sci. 22 349-355 (1999)

6. M. Janz. Materials and Structures. 35 141-148 (2002)

7. J. Martin, M. J. Mosquera, R. Merello. Am. Ceram. Soc. Bull. 75 66-70 (1996)

8. M. J. Mosquera, R. Alcantara, J. Martin. Am. Ceram. Soc. Bull. 77 76-81 (1998)

9. I. Ioannou, A. Hamilton, C. Hall. Cement Concrete Res. 38 766-771 (2008) 